\title{
3D study of plastic flow localization at a void-sheet
}

\section{Tvergaard, Viggo; Legarth, Brian Nyvang}

\section{Published in:}

International Journal of Mechanical Sciences

Link to article, DOI:

10.1016/j.ijmecsci.2020.105426

Publication date:

2020

Document Version

Peer reviewed version

Link back to DTU Orbit

\section{Citation (APA):}

Tvergaard, V., \& Legarth, B. N. (2020). 3D study of plastic flow localization at a void-sheet. International Journal of Mechanical Sciences, 173, [105426]. https://doi.org/10.1016/j.jimecsci.2020.105426

\section{General rights}

Copyright and moral rights for the publications made accessible in the public portal are retained by the authors and/or other copyright owners and it is a condition of accessing publications that users recognise and abide by the legal requirements associated with these rights.

- Users may download and print one copy of any publication from the public portal for the purpose of private study or research.

- You may not further distribute the material or use it for any profit-making activity or commercial gain

- You may freely distribute the URL identifying the publication in the public portal

If you believe that this document breaches copyright please contact us providing details, and we will remove access to the work immediately and investigate your claim. 


\title{
3D study of plastic flow localization at a void-sheet
}

\author{
Viggo Tvergaard and Brian Nyvang Legarth* \\ Department of Mechanical Engineering, Solid Mechanics, Technical university of Denmark \\ DK-2800 Kgs. Lyngby, Denmark \\ *Corresponding author: bnl@mek.dtu.dk
}

\begin{abstract}
A single layer of voids in a ductile material is considered to study the effect of this material imperfection on the onset of shear band localization, and to study the failure evolution in the void-sheet mechanism. The layer is inclined relative to the main tensile direction, and the initially spherical voids are taken to be uniformly spaced both along the inclined direction and transverse to it. The material is elastic-viscoplastic with isotropic hardening. When the solid is subjected to a prescribed strain-rate and the shear band width is negligible compared to specimen dimensions, an infinite ratio of the strain-rate in the band relative to the overall prescribed strain-rate defines the onset of localization. For each stress-state analyzed a limited number of initial inclinations of the void layer are considered, with an angular spacing of 5 degrees, and the angle of inclination giving the smallest critical strain is used as a good approximation of the critical band orientation. Different initial void sizes, relative to the void spacing, are considered. It is shown that increased void spacing in the transverse direction significantly delays localization. Also the effects of different levels of strain hardening are compared. The evolution of the void shape during shear localization is studied, to be able to follow the void-sheet failure mechanism. The critical strain for the onset of void coalescence is compared with the critical strain for shear bands.
\end{abstract}

Keywords: Shear bands, localization, large strain, viscoplasticity, void shape evolution.

\section{Introduction}

In a ductile metal containg voids fracture due to final coalescence of an array of uniformly growing voids is seen in some cases, as at the centre of the neck in a round tensile bar. But in other cases a point of bifurcation away from the uniform state of deformation is reached before coalescence. In studies based on continuum models of dilational plastic deformation Rudnicki and Rice [1] and Yamamoto [2] have shown that the critical strain for shear band instabilities is much reduced by plastic dilatation. For a microporous metal the properties can be represented by the dilational plasticity model of Gurson [3], where bifurcation into a shear band corresponds to loss of ellipticity of the governing differential equations (Yamamoto [2], Tvergaard [4], Saje, Pan and Needleman [5]). Often, localization in a shear band is not initiated at a bifurcation point, but results from an initial imperfection which grows and gradually leads to plastic flow localization, where the plastic deformations continue only inside the band while elastic unloading has occurred outside the band $[2,5]$. In connection with shear bands in porous metals there is much interest in the void-sheet fracture mechanism discussed by Rogers [6], where the voids inside the band continue growing towards coalescence while they are being smeared out in the plastic shear field. Failure in this void-sheet mechanism develops whether or not the shear band initiated from an imperfection or from a bifurcation. The shear localization is often observed in the ligament between two larger voids (Van Stone and Psioda [7], Tvergaard [8]).

There has been much recent interest in studies of ductile failure by void growth to coalescence, e.g. in a recent review by Benzerga et al. [9] where several aspects are discussed. The effect of void shape evolution during ductile failure in a shear field has been analysed by Barsoum and Faleskog [10], Tvergaard [11], Nielsen et al [12], and Morin et al. [13]. The behavior of such voids in a cyclic shear field has been analyzed in [14]. There has also been recent studies of strain gradient effects on void growth to coalescence by Holte et al. [15].

The analysis of shear band development in a homogeneously deformed porous medium, e.g. represented by the Gurson model, makes use of the requirement that there has to be compatibility and equilibrium along the interface to the shear band, but otherwise the material inside the band as well as that outside remains homogeneously deformed on either side of the interface plane. However, if the initial imperfection inside the band is represented in terms of discrete voids there will not be a plane between the inside and outside fields that remains exactly planar, and the fields will not remain homogeneous.

In a plane strain analysis of void-sheet failure Tvergaard [16] considered an imperfection in the form of a row of uniformly spaced circular cylindrical voids, initially inclined to the main tensile direction. At increasing distance from the row of voids the stress and strain fields approach the uniform fields far away from the voids. Therefore, the approximation was made that at a chosen plane well away from the voids compatibility with the uniform far field is prescribed together with equilibrium on the average. In [9] also the presence of a rigid inclusion inside the void and the effect of contact between the inclusion and the void surface after void nucleation are accounted for. The same method was later 
used by Tvergaard and Needleman [17] to study nonlocal effects on localization in a void-sheet and by Tvergaard [18] to study void-sheet failure under stress states near pure shear. A somewhat similar method has been used in 3D analyses by Tekoğlu et al. [19] to study the competition between localization and void coalescence as the main mechanism for initiating ductile fracture and by Barsoum and Faleskog [20].

The present investigation uses a 3D version of the model from [16], with the matrix material around the voids represented as elastic-viscoplastic. Different stress states are considered for the uniformly deformed material outside the band region, for different ratios of the void radius to the void spacing along the inclined band. Also the effect of different transverse void spacing is analysed, as well as the effect of the strain hardening level. For each imperfection considered a number of initial band inclinations are analysed to get an estimate of the lowest critical strain for localization. The analyses make it possible to study the evolution of void shape near the onset of the shear band instability.

\section{Problem Formulation}

The initial imperfection analysed consists of spherical voids with initial radius $R_{0}$ placed on a plane inclined by the angle $\psi_{0}$ relative to the $x_{1}$ axis, with spacing $2 D_{0}$ along the inclined plane and the spacing $2 C_{0}$ in the transverse direction parallel to the $x_{3}$ axis, see Fig. 1. The outer planes limiting the layer analysed numerically are indicated by dashed lines in Fig. 1, with a distance $B_{0}$ from the void plane, when measured parallel to the $x_{2}$ axis.

Most of the numerical studies are carried out by analysing the unit cell shaded in Fig. 1, where $A_{0}=D_{0} \cos \left(\psi_{0}\right)$. On the $x_{3}$ axis the displacements in the $x_{1}$ and $x_{2}$ directions are zero, and in origo, at the void center, all displacements are zero. Also, the tractions on the void surface are zero.

On the two sides at $A D$ and $B C$ the stresses and displacements satisfy periodicity conditions, which are enforced by using a penalty method. On the plane at $A B$ the stresses and displacements satisfy rotational symmetry about the $x_{3}$ axis, which is also enforced by a penalty method. On the planes $x_{3}=0$ and $x_{3}=C_{0}$ symmetry conditions are prescribed. It is noted that if instead symmetry conditions were prescribed on the planes $x_{3}=-C_{0}$ and $x_{3}=C_{0}$, this would give the same result, as these are also planes of symmetry for the solution. Finally, on the plane at $D C$ the displacements relative to those at point $D$ are taken to be fully compatible with those in the uniformly strained solid outside the layer, and the average stresses equal the stresses in the uniformly strained solid.

For the material outside the layer the uniform stress fields and strain fields are first determined in the elasticviscoplastic material with a prescribed strain rate $\dot{\varepsilon}_{22}=\dot{\varepsilon}_{2}^{O}$ in the main tensile direction. In this solution fixed stress ratios are prescribed by specifying the constants $\kappa_{1}$ and $\kappa_{3}$, where $\Sigma_{1}=\kappa_{1} \Sigma_{2}$ and $\Sigma_{3}=\kappa_{3} \Sigma_{2}$ and $\Sigma_{2}>0$. At regular time intervals these stresses and strains are tabulated so that at any time in the numerical solution the values to be used as boundary conditions on the end plane of the unit cell at $D C$ can be found by interpolation in the table. It is noted that the shear band will be very thin compared to the dimensions of the solid, and therefore the strain rate in the material outside the layer will be equal to that applied to the whole solid.

In the material outside the layer the value of the mean stress is $\Sigma_{M}=\frac{1}{3}\left(\Sigma_{1}+\Sigma_{2}+\Sigma_{3}\right)=\frac{1}{3}\left(\kappa_{1}+1+\kappa_{3}\right) \Sigma_{2}$ and the Mises stress is

$$
\begin{aligned}
\Sigma_{e} & =\sqrt{\frac{1}{2}\left[\left(\Sigma_{1}-\Sigma_{2}\right)^{2}+\left(\Sigma_{2}-\Sigma_{3}\right)^{2}+\left(\Sigma_{3}-\Sigma_{1}\right)^{2}\right]} \\
& =\sqrt{\frac{1}{2}\left[\left(\kappa_{1}-1\right)^{2}+\left(1-\kappa_{3}\right)^{2}+\left(\kappa_{3}-\kappa_{1}\right)^{2}\right]} \Sigma_{2}
\end{aligned}
$$

such that the stress triaxiality, $T$, in terms of the stress ratios $\kappa_{1}$ and $\kappa_{3}$ is

$$
T=\frac{\Sigma_{M}}{\Sigma_{e}}=\frac{\sqrt{2}}{3} \frac{\kappa_{1}+1+\kappa_{3}}{\sqrt{\left[\left(\kappa_{1}-1\right)^{2}+\left(1-\kappa_{3}\right)^{2}+\left(\kappa_{3}-\kappa_{1}\right)^{2}\right]}}
$$

The Lode parameter, $L$, is

$$
L=\frac{2 \sigma_{2}-\sigma_{1}-\sigma_{3}}{\sigma_{1}-\sigma_{3}}
$$

where the order is $\sigma_{1} \geq \sigma_{2} \geq \sigma_{3}$. Here, $\Sigma_{2}$ is always the largest, i.e. $\sigma_{1}=\Sigma_{2}$, whereas $\kappa_{1}$ and $\kappa_{3}$ define whether or $\operatorname{not} \Sigma_{1} \geq \Sigma_{3}$.

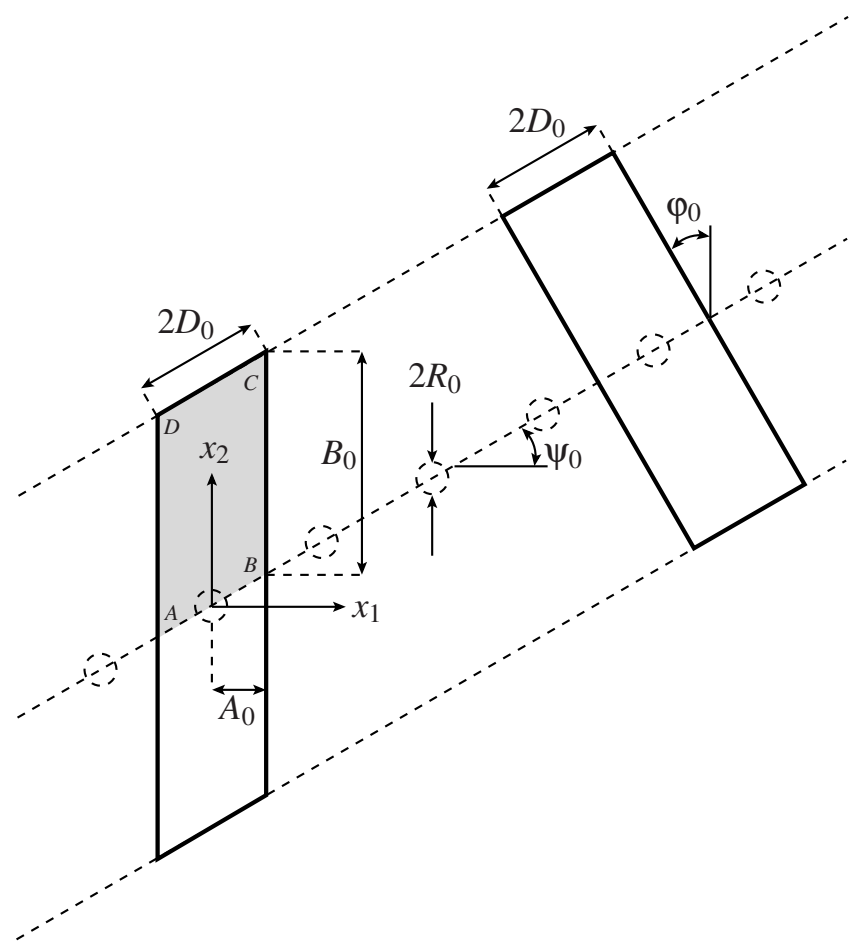

Fig. 1. A planar view of the 3D unit cell, which has the dimension $C_{0}$ in the $x_{3}$ direction. The unit cell shown shaded is used for most computations. Also an alternative unit cell is shown, tilted by an angle $\varphi_{0}$ relative to the $x_{2}$ axis (shown for $\varphi_{0}=\psi_{0}$ ). 
Fig. 1 also shows an alternative unit cell, rotated by an angle $\varphi_{0}$ relative to the $x_{2}$ axis. With the same periodicity conditions and other boundary conditions mentioned above for the shaded unit cell, this alternative unit cell can also be used to solve the localization problem. The important common thing for the two unit cells is that at the top of the cell, at $D C$, full compatibility with the uniform deformation field outside the layer is required, together with equilibrium on the average. Then, for different values of the angle $\varphi_{0}$ the function of the unit cell is simply to determine the stress and strain fields inside the layer.

The elasto-plastic material model used accounts for small elastic but finite plastic deformations in a visco-plastic formulation. The components of the second-order nonsymmetric velocity gradient tensor, $L_{i j} \neq L_{j i}$, are determined by $L_{i j}=\frac{\partial \dot{u}_{i}}{\partial x_{j}}=\dot{u}_{i},{ }_{j}$, where the displacement field components for the material are $u_{i}$ and a superposed dot indicates a time derivative. The symmetric part of $L_{i j}$ is the strain rate, $D_{i j}=D_{j i}$, and the antisymmetric part is the continuum spin tensor, $\omega_{i j}=-\omega_{j i}$. The strain rate is decomposed into elastic and plastic parts as

$$
D_{i j}=D_{i j}^{\mathrm{e}}+D_{i j}^{\mathrm{p}}
$$

where the superscripts e and $\mathrm{p}$ denote the elastic and plastic parts, respectively. The isotropic elasticity relations are assumed to be given by Hooke's law through the constitutive tensor $C_{i j k l}$ determined by Young's modulus, $E$, and Poisson's ratio, $v$.

Assuming normality and a Mises yield surface with $\sigma_{e}=\sqrt{\frac{3}{2} s_{i j} s_{i j}}$, where the stress deviator is $s_{i j}=\sigma_{i j}$ $\frac{1}{3} \delta_{i j} \sigma_{k k}$ and $\sigma_{i j}=\sigma_{j i}$ is the symmetric Cauchy stress, the viscoplastic part of the strain rate is taken to be

$$
D_{i j}^{\mathrm{p}}=\dot{\phi} \frac{3}{2} \frac{s_{i j}}{\sigma_{e}} \quad ; \quad \dot{\phi}=\dot{\varepsilon}_{0}\left(\frac{\sigma_{e}}{g\left(\varepsilon^{\mathrm{p}}\right)}\right)^{1 / m}
$$

where the magnitude of the plastic strain increment is $\dot{\phi}, \dot{\varepsilon}_{0}$ is a reference strain rate, $m$ is a strain rate sensitivity parameter and $g\left(\varepsilon^{\mathrm{p}}\right)=\sigma_{0}\left(1+\frac{\varepsilon^{\mathrm{p}}}{\varepsilon_{0}}\right)^{n}$ is an isotropic deformation dependent power-law hardening function. Here, $\sigma_{0}$ is the initial uniaxial reference stress and $\varepsilon_{0}$ and $n$ are the hardening parameters. The accumulated effective plastic strain, $\varepsilon^{\mathrm{p}}$, is

$$
\varepsilon^{\mathrm{p}}=\int \dot{\varepsilon}^{\mathrm{p}} \mathrm{d} t \quad ; \quad \dot{\varepsilon}^{\mathrm{p}}=\sqrt{\frac{2}{3} D_{i j}^{\mathrm{p}} D_{i j}^{\mathrm{p}}}
$$

The objective rate with respect to $\omega_{i j},\left(^{\nabla}\right)$, of the symmetric Kirchhoff stress, $\tau_{i j}=\tau_{j i}$, is introduced as [21]

$$
\stackrel{\nabla}{\tau}_{i j}=C_{i j k l} D_{k l}^{\mathrm{e}}=C_{i j k l}\left(D_{k l}-D_{k l}^{\mathrm{p}}\right)
$$

such that the rate of the Cauchy stress is

$$
\dot{\sigma}_{i j}=\stackrel{\nabla}{\tau}_{i j}+\omega_{i k} \sigma_{k j}+\sigma_{i k} \omega_{j k}-\sigma_{i j} D_{k k}
$$

It is noted, that $D_{k k}=D_{k k}^{\mathrm{e}} \simeq 0$ due to plastic incompressibility, where $D_{k k}^{\mathrm{p}} \equiv 0$.

For a time-independent elastic-plastic solid flow localization is said to occur when elastic unloading takes place in all the material outside the shear band. In the present analysis for an elastic-viscoplastic solid such strain rate reversal is not allowed for, as a constant positive strain-rate $\dot{\varepsilon}_{2}^{O}$ is prescribed outside the band. In an analysis for localization in an elasticviscoplastic polycrystal Tvergaard and Needleman [22] have defined localization by the occurrence of a very large effective plastic strain rate inside the band, relative to the reference strain rate. Based on the displacement rates in the $x_{1}$ direction at points $A$ and $D$ in Fig. 1 a measure of the rate of shear straining in the band is introduced as

$$
\dot{e}_{s}=\frac{-\dot{u}_{1}^{A}+\dot{u}_{1}^{D}}{A_{0}}
$$

When the rate of increase of the ratio $\dot{e}_{s} / \dot{\varepsilon}_{0}$ grows very large localization occurs, as defined by $\dot{e}_{s} / \dot{\varepsilon}_{0}>10^{4}$.

Based on the change of length of the straight line between $B$ and $C$ in Fig. 1 an average logarithmic strain $\varepsilon_{B C}$ can be calculated. Then, the evolution of the ratio $\varepsilon_{B C} / \varepsilon_{2}$ can be plotted as an estimate of the straining of the cell. The void volume $V$ during the deformation is calculated by numerical integration, so that the evolution of the volume normalized by the initial volume, $V / V_{0}$, can be plotted.

\section{Numerical method}

An updated Lagrangian formulation [21,23] based on the principle of virtual work is used. Disregarding body forces the incremental form of the principle of virtual work in terms of the Kirchhoff stress is [23,24]

$$
\begin{array}{r}
\Delta t \int_{V}\left({ }_{\tau}^{\nabla} \delta D_{i j}-\sigma_{i j}\left(2 D_{i k} \delta D_{k j}-L_{k j} \delta L_{k i}\right)\right) \mathrm{d} V= \\
\Delta t \int_{S} \dot{T}_{i} \delta \dot{u}_{i} \mathrm{~d} S-\left[\int_{V} \sigma_{i j} \delta D_{i j} \mathrm{~d} V-\int_{S} T_{i} \delta \dot{u}_{i} \mathrm{~d} S\right]
\end{array}
$$

where $V$ is the volume and $S$ is the surface, $T_{i}=\sigma_{i j} n_{j}$ are the tractions and $\delta \dot{u}_{i}$ are the virtual velocities, all referred to the current deformed configuration. Therefore, $\tau_{i j}$ is identical to $\sigma_{i j}$. The bracketed terms in Eq. (10) vanish if the current state satisfies equilibrium. However, due to numerical errors the solution tends to drift away from the true equilibrium path, and including the bracketed terms in Eq. (10) as an additional load term, prevents such drifting.

For the numerical finite element solution the cell is discretisized using iso-parametric, quadratic 20 -node brick elements with three translational degrees of freedom per node. 
Reduced $2 \times 2 \times 2$ Gauss integration is adopted within each element. Fig. 2 shows an example of a mesh consisting of 1184 elements (17385 DOFs) for a unit cell specified by $B_{0} / A_{0}=4, C_{0} / A_{0}=1, R_{0} / D_{0}=0.25$ and $\psi_{0}=35^{\circ}$. The elements near the void are made smaller, in order to represent the large deformations expected during void expansion. In each increment, the time step, $\Delta t$, for the next increment is adjusted according to $\left(\dot{\varepsilon}^{\mathrm{p}}\right)_{\text {max }} \cdot \Delta t \leq 10^{-5}$, where $\left(\dot{\varepsilon}^{\mathrm{p}}\right)_{\max }$ is the maximum effective plastic strain rate in any Gauss point.

To compute the finite element solution three subproblems are solved, and in each case the average stresses on the top surface of the cell, at $C D$ in Fig. 1, are determined for the $x_{1}$ and $x_{2}$ directions. Subsequently, the three solutions are superposed to obtain the solution with the correct average stresses on the top surface. In the first sub-problem the deformations and rotations of the top plane are specified as required according to the required compatibility with the uniformly strained solid outside the layer, but the displacement increments at the top plane are chosen small. In the next two sub-problems the deformations and rotations of the top plane are taken to be zero, but the displacement increments of the top plane are given a small specified value, first in the

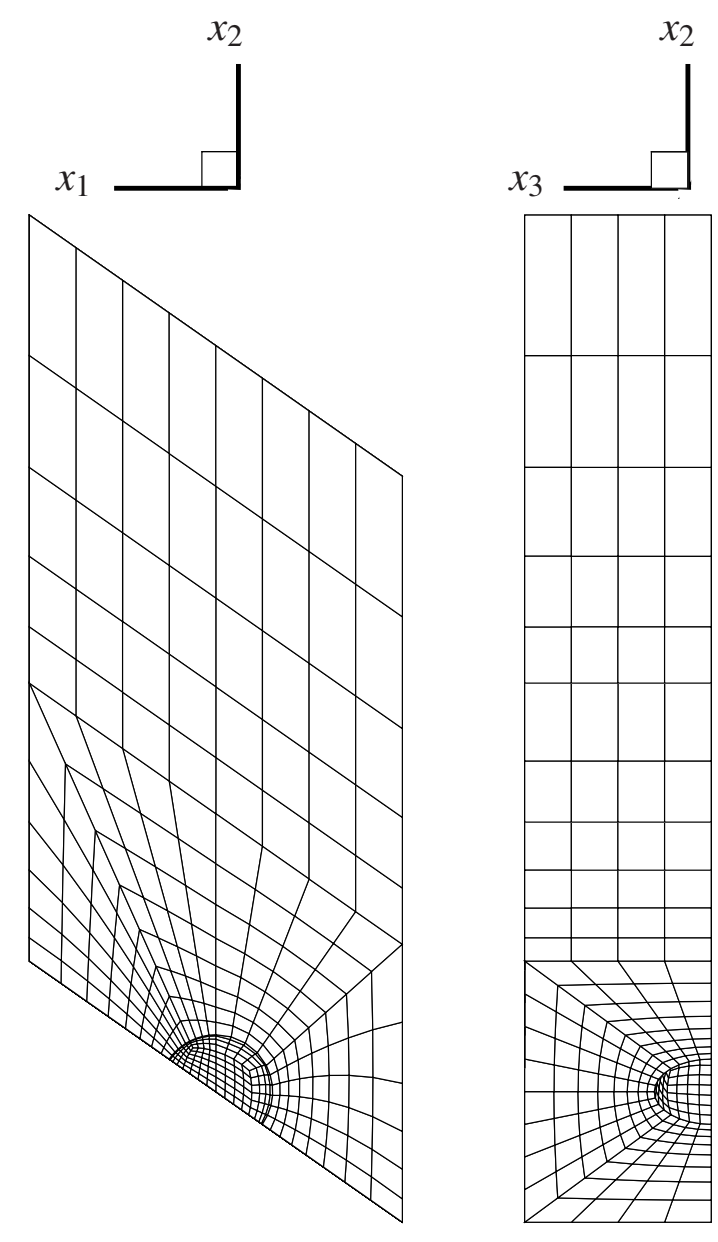

Fig. 2. Example of mesh for $B_{0} / A_{0}=4, C_{0} / A_{0}=1, R_{0} / D_{0}=$ 0.25 and $\psi_{0}=35^{\circ}$. $x_{1}$ direction, and then in the $x_{2}$ direction. Subsequently, the amounts of the two last solutions to be superposed on the solution to the first sub-problem are calculated so that also the average stresses on the top plane are as required according to the uniformly strained solid outside the layer.

\section{Results}

Most of the numerical analyses are carried out for the material parameters $\sigma_{0} / E=0.003$, Poisson's ratio $v=1 / 3$, the strain hardening exponent $n=0.1$, the strain hardening parameter $\varepsilon_{0}=0.002$, the reference strain rate $\dot{\varepsilon}_{0}=0.001 \mathrm{~s}^{-1}$ and the strain rate exponent $m=0.01$. The dimensions of the unit cell are specified by $B_{0}=4 A_{0}$ and $C_{0}=A_{0}$, and the material is taken to be an isotropic Mises solid. In all cases the prescribed strain rate outside the layer is taken to be $\dot{\varepsilon}_{2}^{O}=\dot{\varepsilon}_{0}$.

In Fig. 3 the stress-ratios in the material outside the layer are specified by $\kappa_{1}=0.0$ and $\kappa_{2}=0.5$, so that $T=0.5774$ and $L=0$, corresponding to the stress state in uniaxial plane strain tension. The initial void radius is given by $R_{0} / D_{0}=0.35$. Calculations have been carried out for four different values of the angle of inclination $\psi_{0}$ of the layer containing the imperfection, $25^{\circ}, 30^{\circ}, 35^{\circ}$ and $40^{\circ}$, respectively. Fig. 3a shows the evolution of the void volume, $V / V_{0}$, vs. the straining $\varepsilon_{2}$ outside the layer, for the four angles. Fig. $3 b$ shows the evolution of the relative strain, $\varepsilon_{B C} / \varepsilon_{2}$, and Fig. $3 \mathrm{c}$ shows the measure of the rate of shear straining in the band, $\dot{e}_{s} / \dot{\varepsilon}_{0}$, as given by Eq. (9). It is seen that the ratio $\dot{e}_{S} / \dot{\varepsilon}_{0}$ is small during most of the deformation, but then grows very large at a well-defined strain value, which gives the critical strain for localization. The curves for $V / V_{0}$ show that the void volumes increase some during the localization process, but in fact for the critical layer inclination, $\psi_{0}=35^{\circ}$, where the onset of localization occurs first, the void volume has not reached twice the initial volume.

On the curve for $\psi_{0}=40^{\circ}$ in Fig. 3a the void growth is slower than found for the other three cases, and the growth stops completely at the end of the curve, during shear localization. When localization is reached in this case the current angle of inclination of the layer is $\psi_{c}=50.4^{\circ}$. In many earlier investigations of shear band localization in porous metals (e.g. see Tvergaard [25]) it is characteristic that the localization strain increases rapidly soon after that the value of the current angle of inclination $\psi$ has exceeded $45^{\circ}$, and for slightly larger angles no localization is predicted at all. Thus, it is expected that here $\psi_{0}=40^{\circ}$ is in the range where the localization strain is increasing and that for slightly larger values of $\psi_{0}$ no localization would be predicted at all.

The development of the relative strain $\varepsilon_{B C} / \varepsilon_{2}$ is shown in Fig. $3 b$ for the four different initial inclinations of the layer. In each case the ratio increases to somewhere around 1.4 to 1.8 at localization. However, it should be noticed that the final strain ratio at localization depends on the choice of aspect ratio for the unit cell. If $B_{0} / A_{0}$ was chosen much larger the relative strain $\varepsilon_{B C} / \varepsilon_{2}$ at localization would be close to unity. The unit cell is chosen such that the stress and strain states will become nearly uniform, and approaching 
the stress and strain states specified outside the layer, at a distance of about 0.5 to 1.0 times the void spacing, above the bottom of the cell. Thus, the increase of $\varepsilon_{B C}$ above $\varepsilon_{2}$ is only a result of the shear band forming along the layer of voids, and the void expansion. It is noted that each of the curves in Fig. $3 b$ indicate the localization strain by the very high slope at the end, as is shown most clearly by the curves in Fig. 3c.

For the most critical case in Fig. $3, \psi_{0}=35^{\circ}$, Fig. $4 \mathrm{a}$ shows the deformed unit cell, and the strain contours, at the
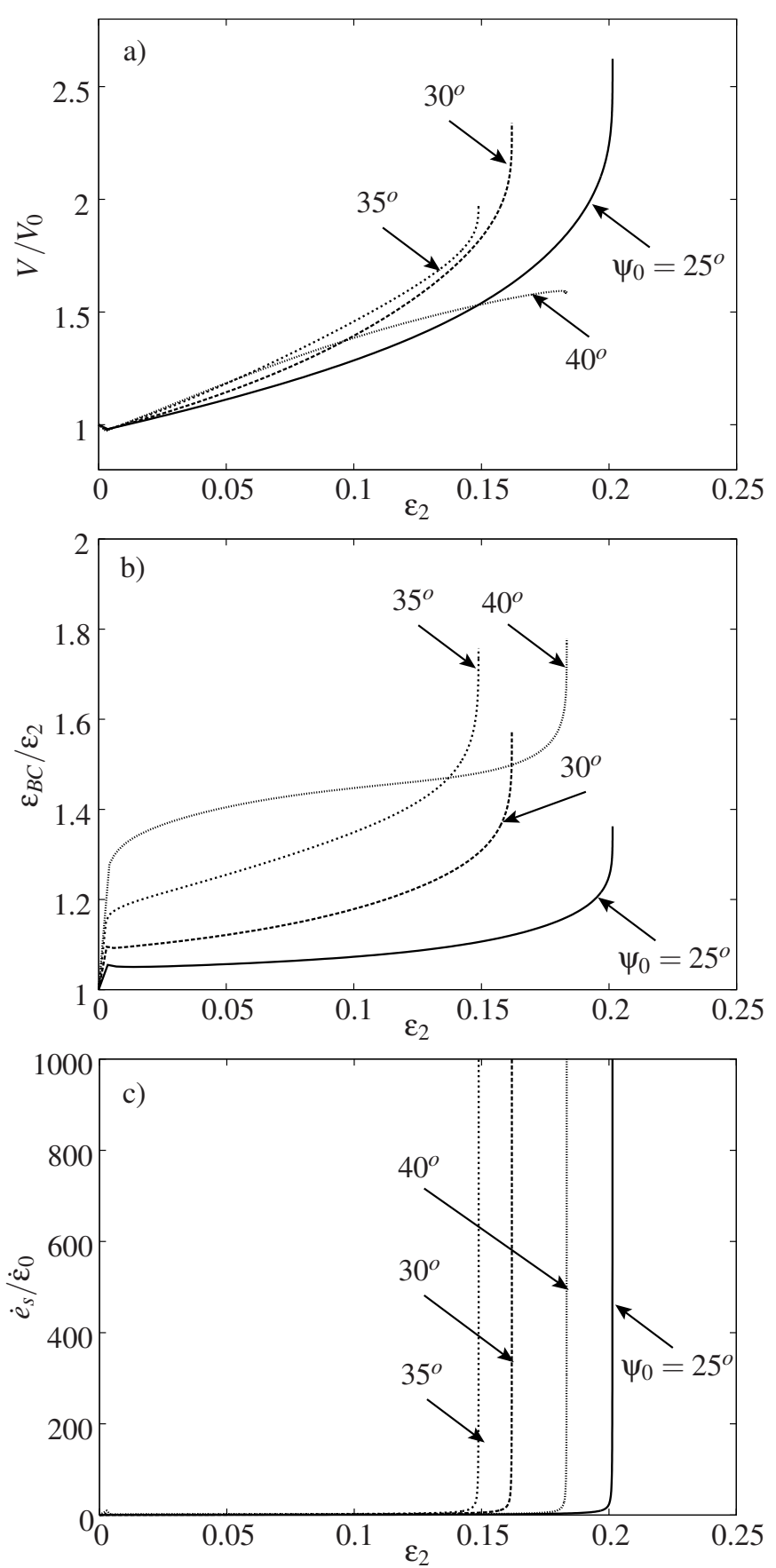

Fig. 3. For $\kappa_{1}=0.0$ and $\kappa_{3}=0.5$ and $R_{0} / D_{0}=0.35$. a) Void volume vs. straining outside the layer. b) Average strain in unit cell vs. straining outside the layer. c) Measure of the rate of shear straining vs. straining outside the layer. end of the computation, just at the onset of shear band localization. Note that the cell is only slightly rotated around the $x_{2}$ axis, therefore in these plots it appears to be much thinner than it actually is. It is seen that the top part of the unit cell has a uniform straining, which is identical to the strain field prescribed in the material outside the layer. Near the void there is a clear shear straining throughout the shear band, and the void is tilting with the shear, while the void volume still grows as seen in Fig. 3a. This tilting of the voids will lead to the characteristic void-sheet fracture surface showing that the voids have been smeared out while they grow to coalescence, as was early observed in experiments by Rogers [6]. Fig. 4b shows the deformed unit cell at the end of the computation for $\psi_{0}=40^{\circ}$ in Fig. 3. Here the void volume has stopped growing, as seen in Fig. 3a, and indeed Fig. 4b illustrates that relative to the shape in Fig. 4a the void has flattened out more relative to the direction along the shear band. As discussed above, the case in Fig. $4 \mathrm{~b}$ is in the range where the localization strain is increasing above the minimum and where no localization would be predicted for a slightly larger value of $\psi_{0}$.

It is noted that the void shape evolution seen in Fig. 4 is somewhat related to the void shape evolution found in shear fields in [11-13]. However, in the shear band considered here there is a tensile stress component across the band, and therefore the void does not completely collapse here, as it does in simple shear.

The effect of using the alternative unit cell in Fig. 1 is illustrated in Fig. 5. The alternative unit cell is chosen to have the tilting angle $\varphi_{0}=25^{\circ}$, but any other value of this angle could have been employed as well. A comparison is here made with the type of unit cell also used for Fig. 3, with $\varphi_{0}=0^{\circ}$. These computations are carried out for $\kappa_{1}=0.0, \kappa_{3}=0.5$, and $\psi_{0}=25^{\circ}$, with a rather large initial void radius given by $R_{0} / D_{0}=0.5$, and the meshes used here are a little cruder than those used for Fig. 3. The figures show the evolution of the void volume, $V / V_{0}$, vs. the straining $\varepsilon_{2}$ outside the layer, Fig. 5a, and of the measure, $\dot{e}_{s} / \dot{\varepsilon}_{0}$, for the rate of shear straining, Fig. 5b. The curves are close enough to make it convincing that the predictions are independent of the value of $\varphi_{0}$, and the agreement would be even closer if the meshes were much finer.

Fig. 6a shows the values of the critical strain for localization $\varepsilon_{2}^{c}$ as a function of the initial angle of inclination $\psi_{0}$ of the layer, as defined by the curves in Fig. 3. The figure also includes localization strains obtained for a higher stress triaxiality, $T=0.8819$ and $L=-0.333$, obtained by specifying $\kappa_{1}=0.25$ and $\kappa_{3}=0.5$. This additional curve has an increased tensile stress in the $x_{1}$ direction, but the same initial void geometry. As expected, the increased stress triaxiality leads to a lower level of the localization strains. Fig. $6 \mathrm{~b}$ shows the same critical localization strains, but now plotted against the current angle of inclination $\psi_{c}$ of the layer in the critical state. On the curve for $\kappa_{1}=0.0$ and $\kappa_{3}=0.5$ the highest current angle shown for localization is $\psi_{c}=50.4^{\circ}$, corresponding to $\psi_{0}=40^{\circ}$, which is a little higher than $45^{\circ}$, as discussed above in relation to Fig. 3a. On the curve for $\kappa_{1}=0.25$ and $\kappa_{3}=0.5$ the localization strain has also been 
calculated for $\psi_{0}=45^{\circ}$.

Computations have been carried out for the double void spacing in the $x_{3}$ direction, $C_{0}=2 A_{0}$, but otherwise the same conditions as considered in Fig. 6 for $\kappa_{1}=0.25$ and $\kappa_{3}=0.5$. This gives a smaller void volume fraction in the layer, which will make the material less prone to shear band localization. Fig. 7a, $7 \mathrm{~b}$ and $7 \mathrm{c}$ show the evolution of the void volume, $V / V_{0}$, of the relative strain, $\varepsilon_{B C} / \varepsilon_{2}$, and of the value of the rate of shear straining in the band, $\dot{e}_{S} / \dot{\varepsilon}_{0}$, vs. the straining $\varepsilon_{2}$ outside the layer, respectively. Among the four initial angles of inclination of the layer all of the figures show that the inclination $\psi_{0}=35^{\circ}$ is the imperfection leading to first localization, at a critical strain only slightly smaller than that found for $\psi_{0}=30^{\circ}$.

Fig. 8a shows the localization strains taken from Fig. 7, vs. the initial angles of inclination of the layer. For comparison the figure includes the corresponding curve for $C_{0}=A_{0}$, which was also shown in Fig. 6. Another way of reducing the imperfection size is to consider smaller voids, $R_{0} / D_{0}=0.25$, still for $C_{0}=A_{0}$. As illustrated in Fig. 8a this also gives much increased localization strains, but not quite as high as those taken from Fig. 7. As a further comparison in Fig. 8a a curve is shown for the smaller void subject to larger transverse stresses, as specified by $\kappa_{1}=0.6$ and $\kappa_{3}=0.5$, corresponding to $T=1.528$ and $L=-0.6$. This results in a low level of localization strains, with the minimum at $\psi_{0}=40^{\circ}$.

In Fig. 8b the critical localization strains from Fig. 8a are plotted against the current angle of inclination $\psi_{c}$ in the critical state, which will be the angle of the final fracture surface. In the cases where the localization strains are relatively high, it is seen that the imperfection layer has rotated more before reaching the state of void-sheet fracture.

The deformed unit cell in Fig. 9 corresponds to the end of the computation in Fig. 8 for $\kappa_{1}=0.25, \kappa_{3}=0.5$, $R_{0} / D_{0}=0.25, C_{0}=A_{0}$ and $\psi_{0}=35^{\circ}$. Here the void is initially smaller than those in Figs. $4 \mathrm{a}$ and $4 \mathrm{~b}$, which tends to give later localization, but also the stress triaxiality is higher, which tends to give earlier localization. As in the previous figures there is a clear shear straining throughout the shear band, and the void is tilting with the shear, but due to the tensile stress in the $x_{1}$ direction the void is less flattened out relative to the direction along the shear band.

Predictions for axisymmetric stress states outside the layer are shown in Fig. 10, where in all cases the value of the Lode parameter is $L=-1.0$. For $\kappa_{1}=\kappa_{3}=0.25, T=0.667$, with $R_{0} / D_{0}=0.35$, the localization strains are relatively high, and it is seen that the minimum localization strain is found for $\psi_{0}=35^{\circ}$. For $\kappa_{1}=\kappa_{3}=0.40, T=1.0$, with $R_{0} / D_{0}=0.35$, the localization strains are much lower, and here the lowest value is found for $\psi_{0}=40^{\circ}$. The computation was also carried out for $\psi_{0}=45^{\circ}$, which gave a slightly higher localization strain. For the same void size and stress state, but with the double spacing in the $x_{3} \mathrm{di}$ rection, $C_{0}=2 A_{0}$, localization predictions are also shown in Fig. 10. Reducing the imperfection size by doubling the spacing in the $x_{3}$ direction gives a big increase of the localization strain, as was also found in Fig. 8. In this case the first critical localization occurs for $\psi_{0}=30^{\circ}$. Also a smaller void,
$R_{0} / D_{0}=0.25$ for $C_{0}=A_{0}$, with higher transverse stresses $\kappa_{1}=\kappa_{3}=0.50, T=1.333$, has been considered, leading to rather small localization strains with the smallest value at $\psi_{0}=40^{\circ}$.

For the axisymmetric stress state, $\kappa_{1}=\kappa_{3}=0.50$, $R_{0} / D_{0}=0.25$ and $\psi_{0}=40^{\circ}$ in Fig. 10 the deformed unit cell at the onset of localization is illustrated in Fig. 11. Here the stress triaxiality is even higher than that in Fig. 9 and the localization condition is reached at a smaller strain. Also, while shearing in the void region is visible in the figure, the void shape has stayed closer to spherical due to the high triaxiality.

Fig. 12 shows localization strains calculated for the stress state $\kappa_{1}=0.5$ and $\kappa_{3}=1.0$. The lower curve corresponds to the void size $R_{0} / D_{0}=0.35$. while the top curve corresponds to the smaller void $R_{0} / D_{0}=0.25$. In both cases localization occurs at small strains, due to the high stress triaxiality. For the smaller void the smallest localization strain found is that for $\psi_{0}=40^{\circ}$, while for the larger void it is $\psi_{0}=45^{\circ}$.

To test the effect of the strain hardening exponent two curves are shown in Fig. 13 for $n=0.2$, both for the void size $R_{0} / D_{0}=0.35$. For $\kappa_{1}=0.25$ and $\kappa_{3}=0.50$, corresponding to $T=0.7599$ and $L=-0.333$, the localization strains are rather high, and the first critical angle of inclination is $\psi_{0}=30^{\circ}$, closely followed by localization at $\psi_{0}=25^{\circ}$ and at $\psi_{0}=35^{\circ}$. When both transverse stress components are doubled, $\kappa_{1}=0.50$ and $\kappa_{3}=1.0(T=1.667$ and $L=1.0)$ the localization strains are much reduced, and the first critical initial inclination is $\psi_{0}=35^{\circ}$. Comparing with Fig. 8 for $\kappa_{1}=0.25$ and $\kappa_{3}=0.50$ it is seen that higher strain hardening exponent in Fig. 13 gives a significant increase in the critical strain. Also comparing with the curve for $R_{0} / D_{0}=0.35$ in Fig. 12 it is seen that higher hardening increases the localization strain noticeably.

The value of the strain hardening parameter, $\varepsilon_{0}=0.002$, has been used so far. To test the influence of this parameter the curves in Fig. 13 have been recomputed using the larger value, $\varepsilon_{0}=0.004$. When looking at the uniaxial stress-strain curve for the same strain-rate it is seen that this smaller value of $\varepsilon_{0}$ affects the shape of the curve and gives a small reduction of the strain hardening. But the corresponding reduction of the localization strains in Fig. 13 is so small that it would be hardly visible, if plotted.

For $\psi_{0}=0^{\circ}$ axisymmetric unit cell models have been used in $[26,27]$ to show that with time-independent plasticity the onset of coalescence occurs when elastic unloading takes place in all of the unit cell apart from the region just around the void. In the context of the present elastic-viscoplastic model this corresponds to the situation where the curve for the relative strain, $\varepsilon_{B C} / \varepsilon_{2}$ vs. time reaches an infinite slope, analogous to that shown in Fig. $3 \mathrm{~b}$ and Fig. 7b. This analysis has been carried out for $\kappa_{1}=0.25$ and $\kappa_{3}=0.5$ with $R_{0} / D_{0}=0.35$, corresponding to one of the lower curves in Figs. 8a and 8b. Fig. 14 shows the deformed unit cell at the end of the computation, where plastic flow localization and the onset of void coalescence is predicted at the critical strain $\varepsilon_{2}^{c}=0.292$. It is noted that this critical strain value is much 
larger than the corresponding first critical value $\varepsilon_{2}^{c}=0.0565$ found in Figs. 8a and $8 \mathrm{~b}$ for $\psi_{0}=40^{\circ}$. This illustrates that the criterion for the onset of void coalescence in [26] is also valid in a non-axisymmetric case. In addition, the analysis has been carried out for one of the cases with an axisymmetric stress state, for $\kappa_{1}=\kappa_{3}=0.4$ and $R_{0} / D_{0}=0.35$, leading to the critical strain $\varepsilon_{2}^{c}=0.208$ for void coalescence. Again, this critical strain value is much larger than the corresponding first critical value $\varepsilon_{2}^{c}=0.0581$ found in Figs. $8 \mathrm{a}$ and $8 \mathrm{~b}$ for $\psi_{0}=40^{\circ}$. It is noted that also computations representing voids in terms of the Gurson model, e.g. see [25], tend to indicate a much higher strain for plastic flow localization at $\psi_{0}=0^{\circ}$ than at the angle first critical for shear band localization.

In [19] the onset of void coalescence is defined as the point in the deformation history where the ratio of the maximum to the minimum effective plastic strain rate at the void surface first exceeds 15 . For $\psi_{0}=0^{\circ}$ this point is reached earlier than the coalescence point predicted by the criterion in [26]. For values of $\psi_{0}$ in the range where shear band instabilities occur, the final void-sheet failure in the shear band involves void coalescence. As was already observed by Rogers [6], the voids inside the band continue growing towards coalescence while they are being smeared out in the plastic shear field. The beginning of this smear out coalescence process is clearly seen in Figs. 4 and 9 at the point where shear band localization starts.

Localization in shear bands can also occur without any voids in the material, as shown in [22] for crystal plasticity, where a vertex forms on the yield surface. Such shear band formation without the presence of voids is also predicted by $J_{2}$ corner theory [28] as illustrated in [29,30].

\section{Discussion}

The present paper extends the method for shear band analysis proposed in [16] to study the effect of an imperfection in the form of a 3D layer of voids. The material region containing the voids is made sufficiently wide so that the numerically resolved stress and strain states near the outer edges of the region get to agree closely with the fields specified in the uniform material outside the region.

The parametric study has shown how the predicted localization strains depend on the void size relative to spacing along the shear direction, and on an increased spacing transverse to the shear direction. Also on the stress state specified for the material outside the void layer, and on the strain hardening level. The specified stress states include both situations near plane strain, axisymmetric stress states, and other stress states. For each set of parameters considered the possibility of shear band localization has only been analysed for a limited number of initial angles of inclination of the layer of voids, with a spacing of $5^{\circ}$ between the inclinations considered. This is sufficiently close to get a good estimate of the lowest critical strain for localization.

The top curve in Fig. 6 for uniaxial plane strain tension with $T=0.577$ gives the critical strain $\varepsilon_{2}^{c}=0.149$. This can be compared with the second highest curved in Fig. 10, for the same void size, where under axisymmetric conditions the critical strain is higher, $\varepsilon_{2}^{c}=0.211$, even though the stress triaxixality is a little higher, $\mathrm{T}=0.667$. This illustrates the known tendency that axisymmetric stress states give later localization than plane strain.

The effect of double void spacing in the transverse direction, $C_{0}=2 A_{0}$, has been compared with the usual void spacing, $C_{0}=A_{0}$, in both Figs. 8 and 10. With the double spacing the voids have to enforce shearing in much more undamaged material between two neighbouring inclined rows of voids. In both figures it is seen that this results in a significant delay of shear band localization.

In the present elastic-viscoplastic analyses the strain rate exponent has been taken to be $m=0.01$. It is know that this will delay the onset of localization a little. This has been seen in recent analyses of cavitation instabilities [31]. Also analyses of sheet necking [32] have shown such a delay.

With the initially spherical voids it has been possible to follow the shape evolution near the stage where the shear band instability is reached, and it has been shown that the void is flattening out relative to the direction along the shear band, while it is simultaneously rotating with the shear. At the same time the void volume is growing leading towards the void-sheet fracture mechanism, where the voids are smeared out along the shear band while they grow to coalescence. It has also been illustrated that for a band inclination slightly larger than that critical for shear bands the void flattens out more and the void volume stops growing.

With the method for shear band analysis proposed in [16] the unit cell has mostly been taken parallel to the main tensile direction. A number of tilted unit cells are equally useful for the analysis, which has been illustrated by comparing the results obtained by two different unit cells with different angles of tilting relative to the main tensile direction.

Also for $\psi_{0}=0^{\circ}$ a plastic flow localization is predicted, which corresponds to the criterion used by Koplik and Needleman [26] for calculating the onset of void coalescence. Both for an axisymmetric stress state and for a non-axisymmetric stress state calculations using the present model with $\psi_{0}=0^{\circ}$ have been carried out. In both cases it has been found that the onset of void coalescence, as defined by the criterion in [26], is predicted at a significantly higher strain than the corresponding first critical strain for shear band localization.

Here, isotropic hardening has been considered, but it is known from previous analyses of shear band localization that kinematic hardening would tend to predict earlier localization than that found by isotropic hardening. Comparison with localization predictions by $J_{2}$ corner theory of plasticity $[28,29]$ has shown that the higher curvature of the yield surface in kinematic hardening has an effect like that of a rounded vertex. This effect of a rounded vertex has also been shown for a kinematic hardening version of a porous ductile material model [33].

An initial plastic anisotropy in the material around the voids would typically have a strong effect on void growth predictions [34], and is expected to have a strong influence 
on localization predictions as those carried out here. Further results on that require elaborate studies. Some such studies are being carried out now.

\section{References}

[1] Rudnicki, J., and Rice, J., 1975. "Conditions for localization of deformation in pressure-sensitive dilatant materials". Journal of the Mechanics and Physics of Solids, 23(6), pp. 371-394.

[2] Yamamoto, H., 1978. "Conditions for shear localization in the ductile fracture of void-containing materials". International Journal of Fracture, 14(4), pp. 347365 .

[3] Gurson, A., L., 1977. "Continuum theory of ductile rupture by void nucleation and growth: Part I Yield criteria and flow rules for porous ductile media". Journal of Engineering Materials and Technologytransactions of the ASME, 99(1), pp. 2-15.

[4] Tvergaard, V., 1981. "Influence of voids on shear band instabilities under plane strain conditions". International Journal of Fracture, 17(4), pp. 389-407.

[5] Saje, M., Pan, J., and Needleman, A., 1982. "Void nucleation effects on shear localization in porous plastic solids". International Journal of Fracture, 19(3), pp. $163-182$.

[6] Rogers, H., 1960. "Tensile fracture of ductile metals". Metallurgical Society of American Institute of Mining, Metallurgical and Petroleum Engineers - Transactions, 218(3), pp. 498-506.

[7] Stone, R. H., and Psioda, J. A., 1975. "Discussion of metallurgical factors affecting fracture toughness of aluminum alloys". Metallurgical Transactions A, 6(4), pp. 668-670.

[8] Tvergaard, V., 1982. "Ductile fracture by cavity nucleation between larger voids". Journal of the Mechanics and Physics of Solids, 30(4), pp. 265-286.

[9] Benzerga, A. A., Leblond, J. B., Needleman, A., and Tvergaard, V., 2016. "Ductile failure modeling". International Journal of Fracture, 201(1), pp. 29-80.

[10] Barsoum, I., and Faleskog, J., 2007. "Rupture mechanisms in combined tension and shear - Micromechanics". International Journal of Solids and Structures, 44(17), pp. 5481-5498.

[11] Tvergaard, V., 2008. "Shear deformation of voids with contact modelled by internal pressure". International Journal of Mechanical Sciences, 50(10-11), pp. 14591465.

[12] Nielsen, K. L., Dahl, J., and Tvergaard, V., 2012. "Collapse and coalescence of spherical voids subject to intense shearing: studied in full 3D". International Journal of Fracture, 177(2), pp. 97-108.

[13] Morin, L., Leblond, J. B., and Tvergaard, V., 2016. "Application of a model of plastic porous materials including void shape effects to the prediction of ductile failure under shear-dominated loadings". Journal of the Mechanics and Physics of Solids, 94, pp. 148-166.

[14] Nielsen, K. L., Andersen, R. G., and Tvergaard, V.,
2018. "Void coalescence mechanism for combined tension and large amplitude cyclic shearing". Engineering Fracture Mechanics, 189, pp. 164-174.

[15] Holte, I., Niordson, C. F., Nielsen, K. L., and Tvergaard, V., 2019. "Investigation of a gradient enriched Gurson-Tvergaard model for porous strain hardening materials". European Journal of Mechanics - A/Solids, 75, pp. 472-484.

[16] Tvergaard, V., 1989. "Numerical study of localization in a void-sheet". International Journal of Solids and Structures, 25(10), pp. 1143-1156.

[17] Tvergaard, V., and Needleman, A., 1997. "Nonlocal effects on localization in a void-sheet". International Journal of Solids and Structures, 34(18), pp. 22212238.

[18] Tvergaard, V., 2015. "Study of localization in a voidsheet under stress states near pure shear". International Journal of Solids and Structures, 75-76, pp. 134-142.

[19] Tekoğlu, C., Hutchinson, J. W., and Pardoen, T., 2015. "On localization and void coalescence as a precursor to ductile fracture". Philosophical Transactions of the Royal Society A: Mathematical, Physical and Engineering Sciences, 373(2038), pp. 1-19.

[20] Barsoum, I., and Faleskog, J., 2011. "Micromechanical analysis on the influence of the lode parameter on void growth and coalescence". International Journal of Solids and Structures, 48(6), pp. 925-938.

[21] Yamada, Y., and Sasaki, M., 1995. "Elastic-plastic large deformation analysis program and lamina compression test". International Journal of Mechanical Sciences, 37(7), pp. 691-707.

[22] Tvergaard, V., and Needleman, A., 1993. "Shear-band development in polycrystals". Proceedings of the Royal Society of London Series A-mathematical Physical and Engineering Sciences, 443(1919), pp. 547-562.

[23] McMeeking, R. M., and Rice, J. R., 1975. "Finiteelement formulations for problems of large elasticplastic deformation". International Journal of Solids and Structures, 11, pp. 601-616.

[24] Legarth, B. N., 2007. "Strain-gradient effects in anisotropic materials". Modelling and Simulation in Materials Science and Engineering, 15, pp. S71-S81.

[25] Tvergaard, V., 1987. "Effect of yield surface curvature and void nucleation on plastic-flow localization". Journal of the Mechanics and Physics of Solids, 35(1), pp. 43-60.

[26] Koplik, J., and Needleman, A., 1988. "Void growth and coalescence in porous plastic solids". International Journal of Solids and Structures, 24(8), pp. 835-853.

[27] Tvergaard, V., 1982. "On localization in ductile materials containing spherical voids". International Journal of Fracture, 18(4), pp. 237-252.

[28] Christoffersen, J., and Hutchinson, J., 1979. "A class of phenomenological corner theories of plasticity". Journal of the Mechanics and Physics of Solids, 27(5-6), pp. 465-487.

[29] Hutchinson, J., and Tvergaard, V., 1981. "Shear band formation in plane-strain". International Journal of 
Solids and Structures, 17(5), pp. 451-470.

[30] Tvergaard, V., Needleman, A., and Lo, K. K., 1981. "Flow localization in the plane strain tensile test". Journal of the Mechanics and Physics of Solids, 29(2), pp. 115-142.

[31] Tvergaard, V., and Legarth, B. N., 2019. "Effects of anisotropy and void shape on cavitation instabilities". International Journal of Mechanical Sciences, 152, pp. 81-87.

[32] Needleman, A., and Tvergaard, V., 1984. "Limits to formability in rate-sensitive metal sheets". In Mechanical Behaviour of Materials - IV. Proceedings of the Fourth International Conference, J. Carlsson and N. G. Ohlson, eds., Pergamon Press, pp. 51-65, vol.1.

[33] Tvergaard, V., and van der Giessen, E., 1991. "Effect of plastic spin on localization predictions for a porous ductile material". Journal of the Mechanics and Physics of Solids, 39(6), pp. 763-781.

[34] Legarth, B. N., and Tvergaard, V., 2018. "Effects of plastic anisotropy and void shape on full threedimensional void growth". Journal of Applied Mechanics, 85(5), p. 051007. a)

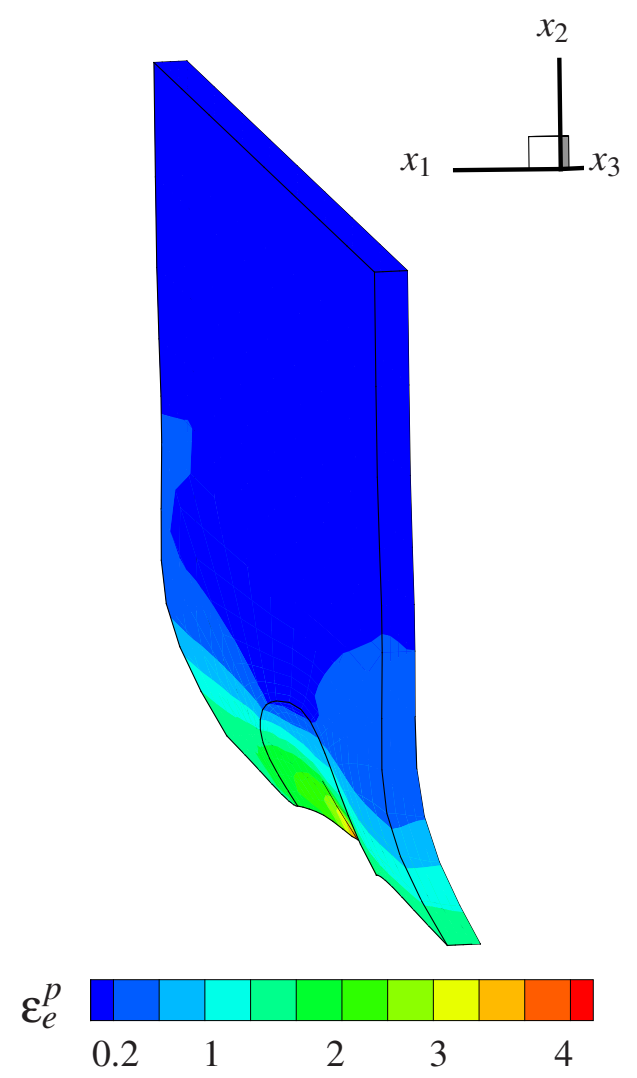

b)

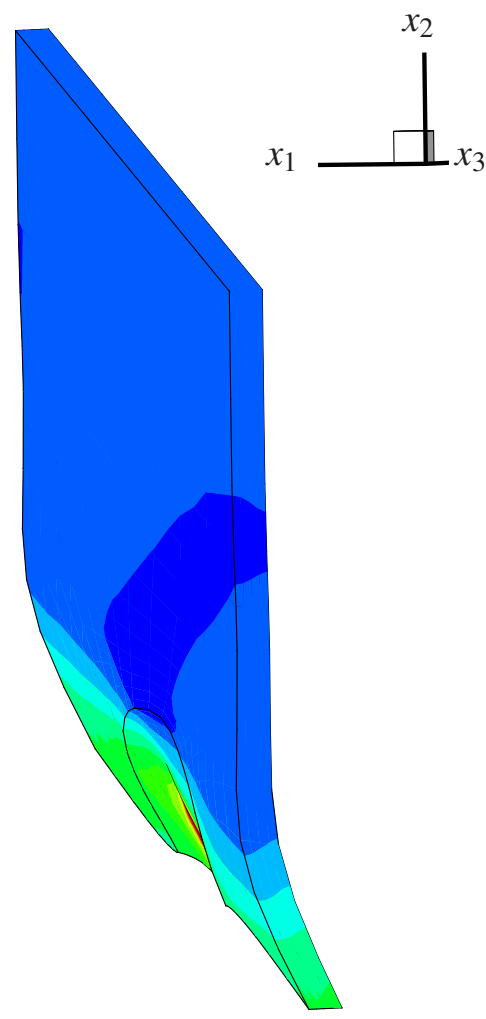

Fig. 4. Deformed unit cell at the end of the computation with $\kappa_{1}=0.0$ and $\kappa_{3}=0.5$, see Fig. 3. a) $\psi_{0}=35^{\circ}$. b) $\psi_{0}=40^{\circ}$. 

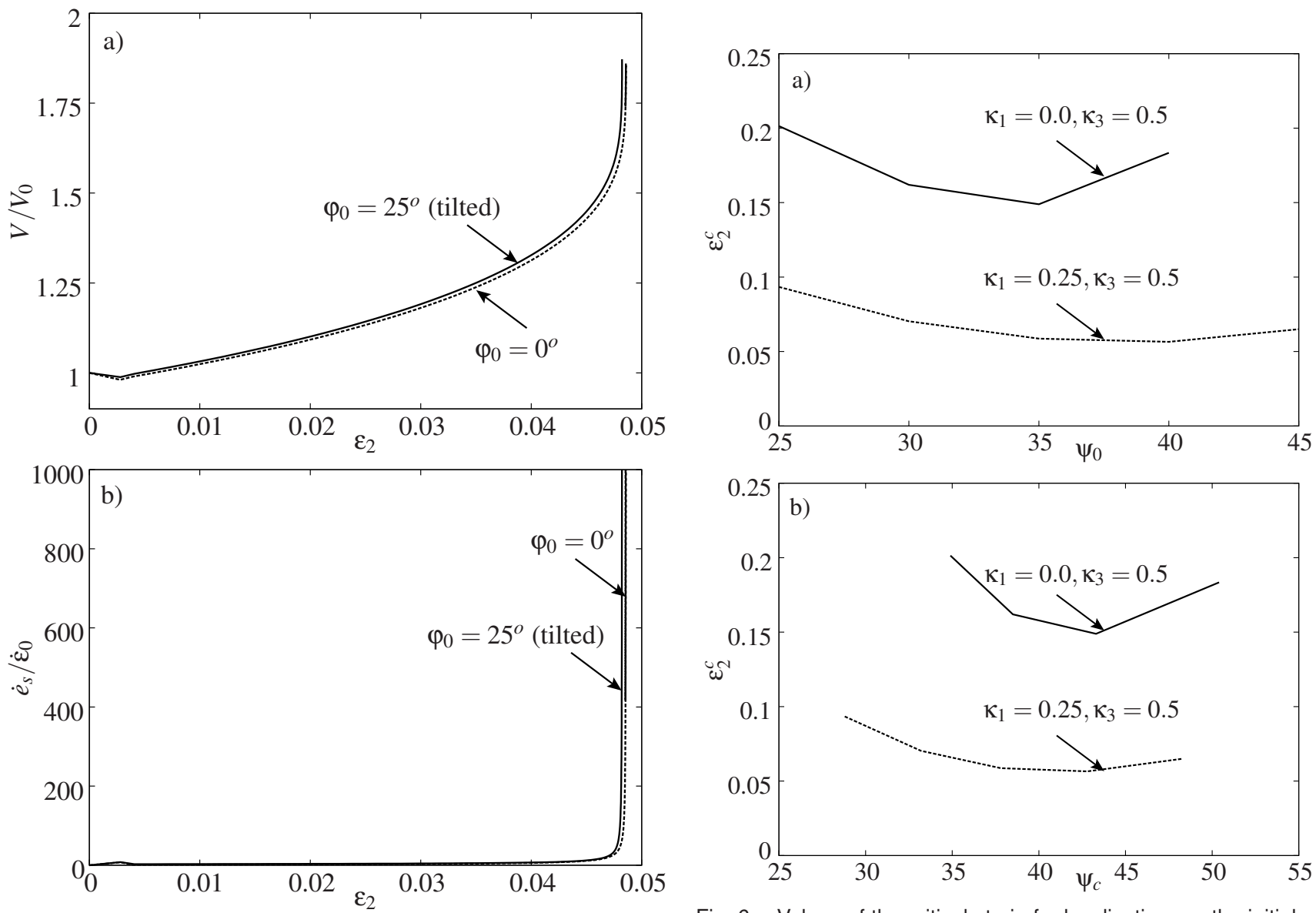

Fig. 5. Comparison with tilted unit cell for $\kappa_{1}=0.0, \kappa_{3}=$ $0.5, \psi_{0}=25^{\circ}$ and $R_{0} / D_{0}=0.5$. a) Void volume vs. straining outside the layer. b) Measure of the rate of shear straining vs. strain-

Fig. 6. Values of the critical strain for localization vs. the initial and current angle of inclination of the layer for $R_{0} / D_{0}=0.35$. a) Initial angle. b) Current angle. 

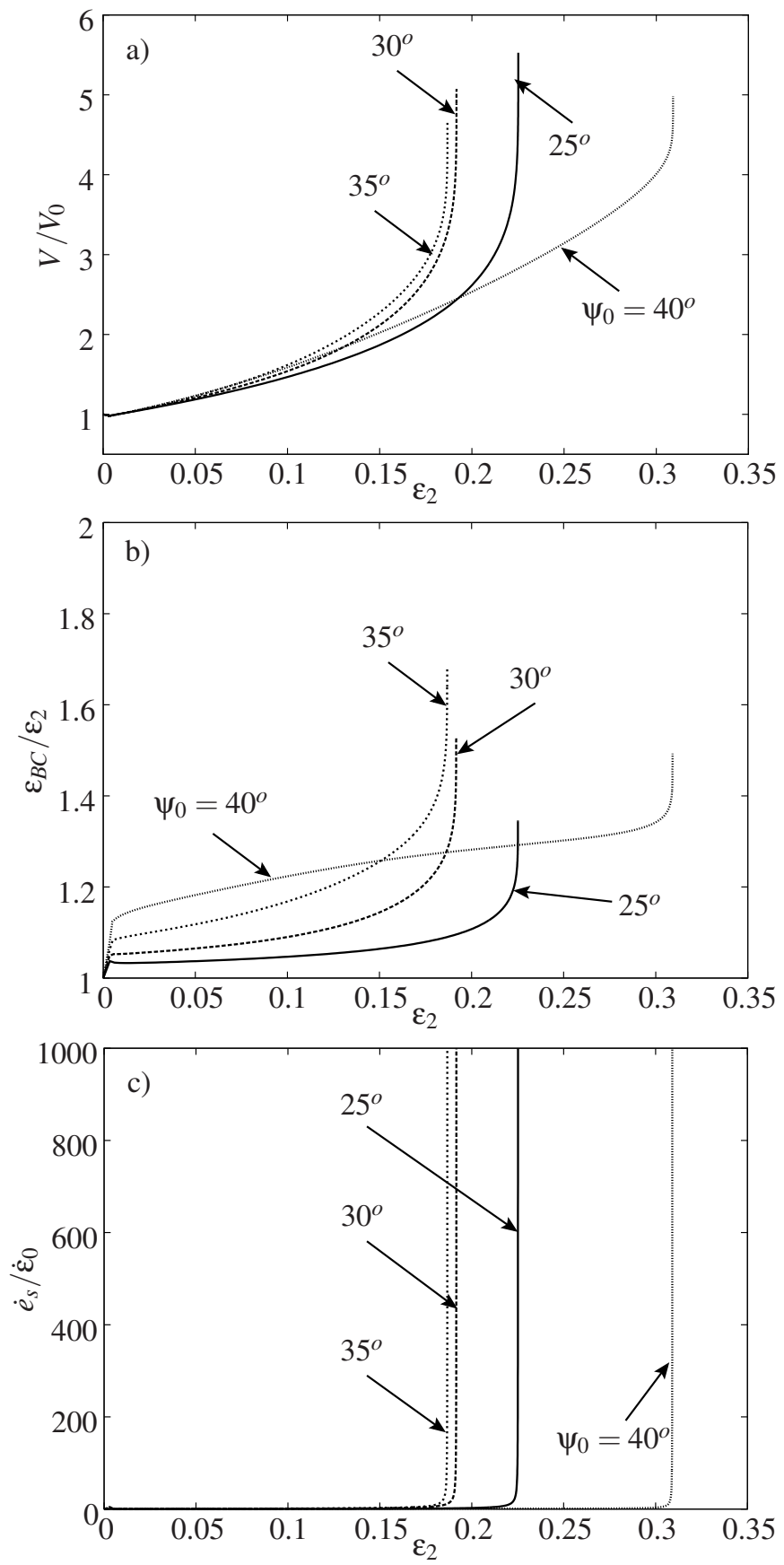

Fig. 7. For $\kappa_{1}=0.25$ and $\kappa_{3}=0.5$ and $R_{0} / D_{0}=0.35$ and double void spacing in the $x_{3}$ direction, $C_{0}=2 A_{0}$. a) Void volume vs. straining outside the layer b) Average strain in unit cell vs. straining outside the layer. c) Measure of the rate of shear straining vs. straining outside the layer.
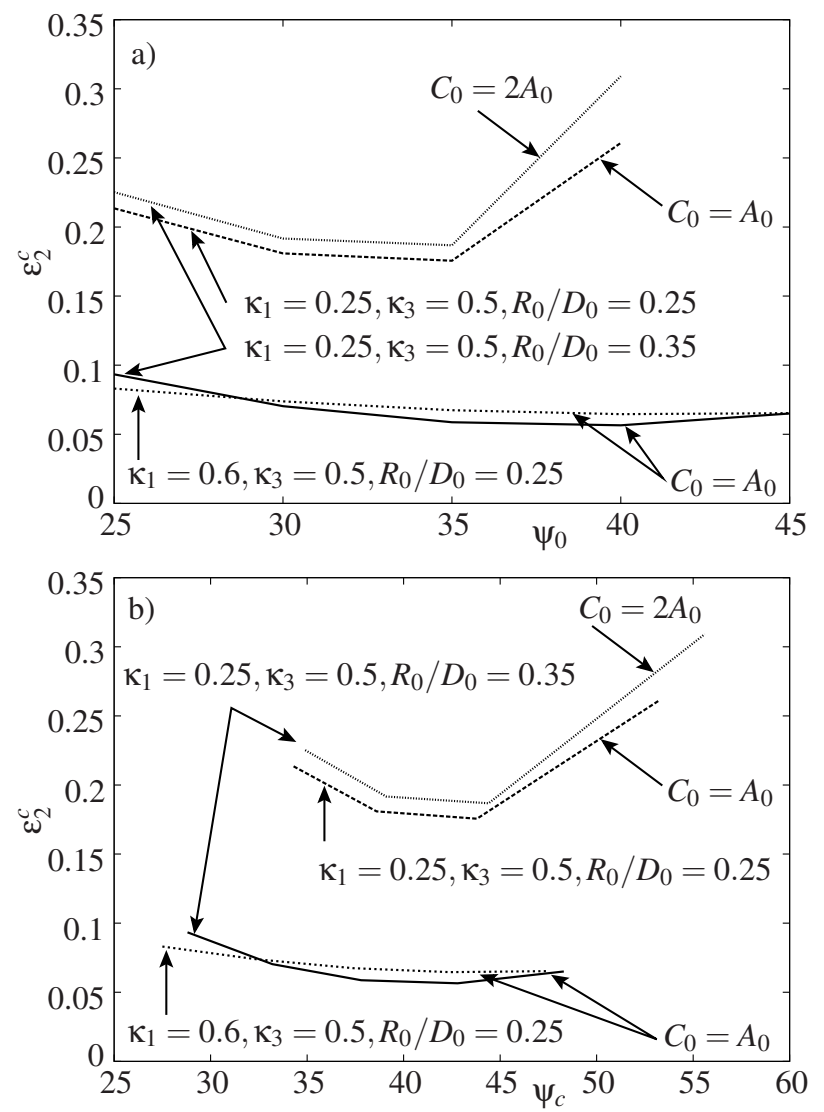

Fig. 8. Values of the critical strain for localization vs. the initial and current angle of inclination of the layer. a) Initial angle. b) Current angle. 


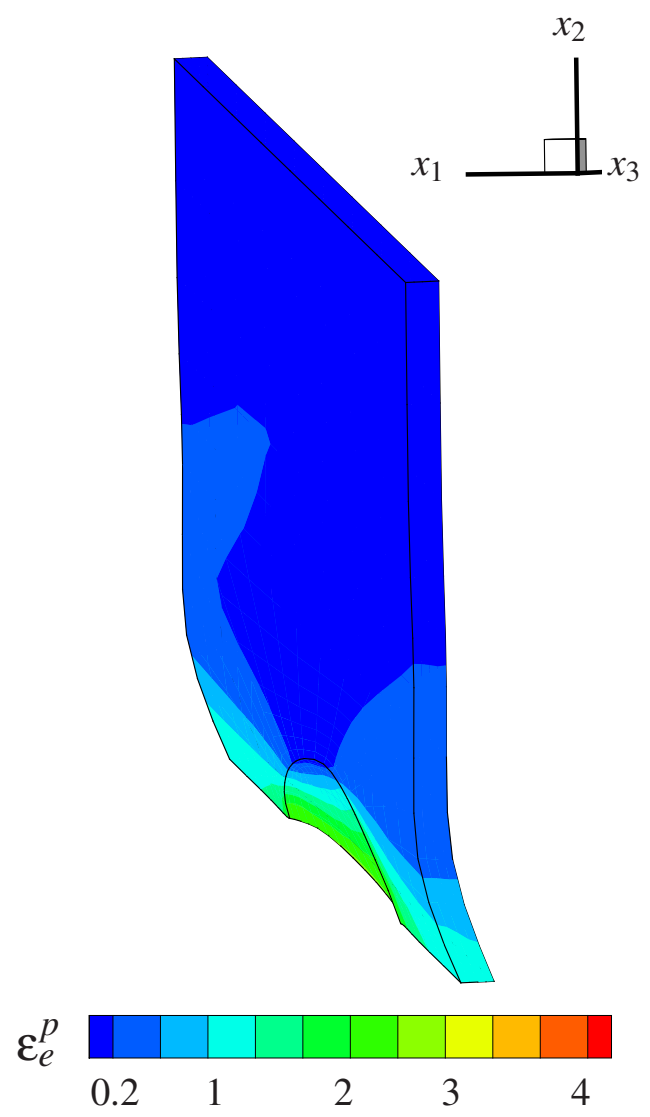

Fig. 9. Deformed unit cell at the end of the computation for $\psi_{0}=$ $35^{\circ}$, with $\kappa_{1}=0.25$, and $\kappa_{3}=0.5$ and $R_{0} / D_{0}=0.25$, see Fig 8.

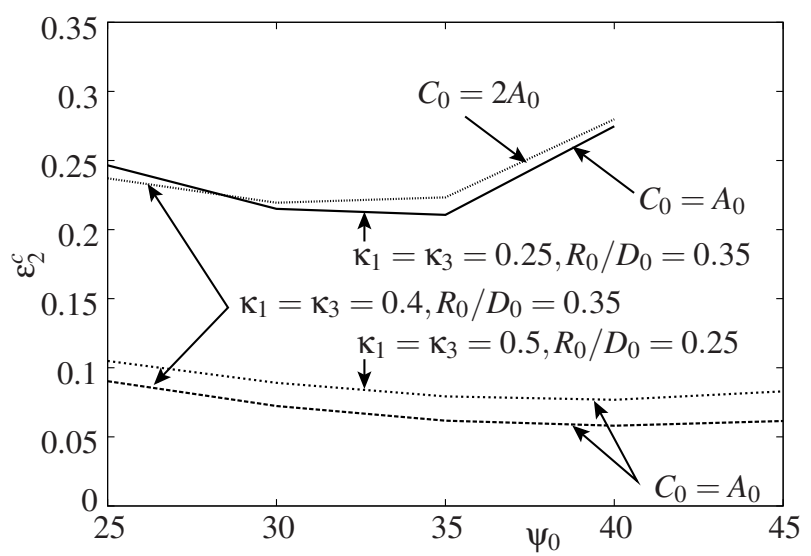

Fig. 10. Values of the critical strain for localization vs. the initial and current angle of inclination of the layer. a) Initial angle. b) Current angle.

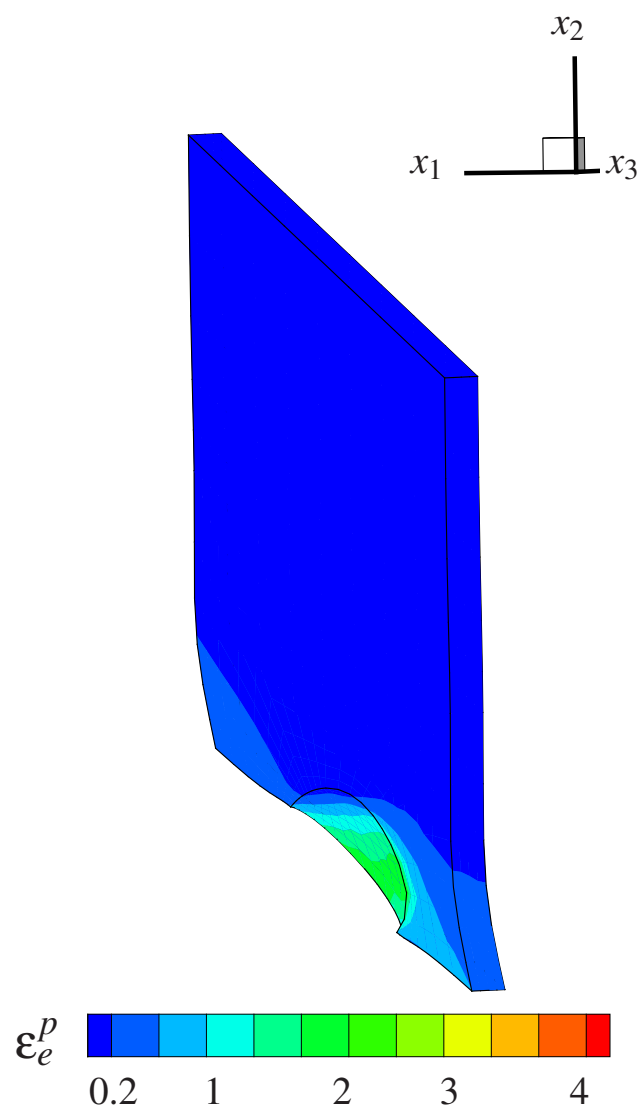

Fig. 11. Deformed unit cell at the end of the computation for $\psi_{0}=$ $40^{\circ}$, with $\kappa_{1}=\kappa_{3}=0.5$ and $R_{0} / D_{0}=0.25$, see Fig. 10.

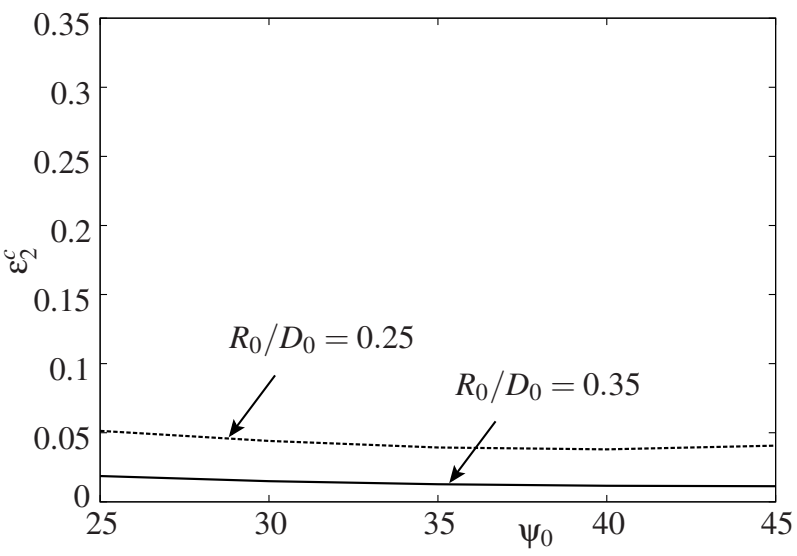

Fig. 12. Values of the critical strain for localization vs. the initial angle of inclination of the layer in cases where $\kappa_{1}=0.50$ and $\kappa_{3}=$ 1.0 . 


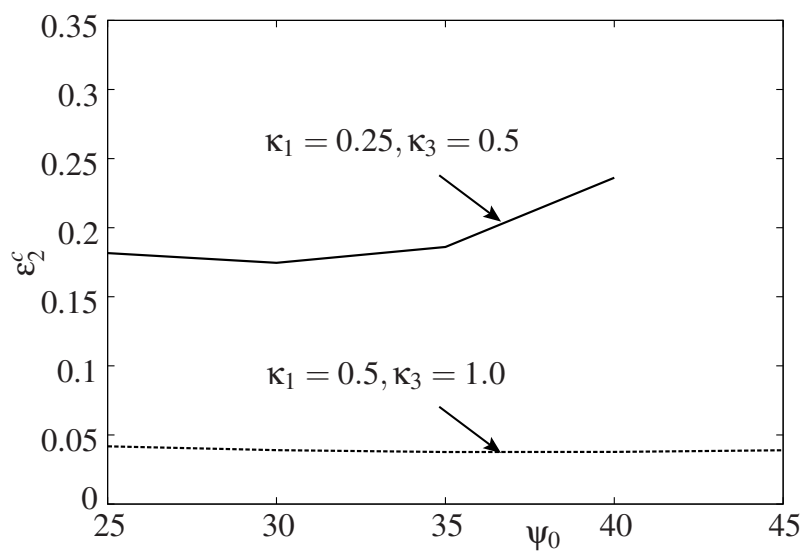

Fig. 13. Values of the critical strain for localization vs. the initial angle of inclination of the layer for $R_{0} / D_{0}=0.35$ and $n=0.2$.

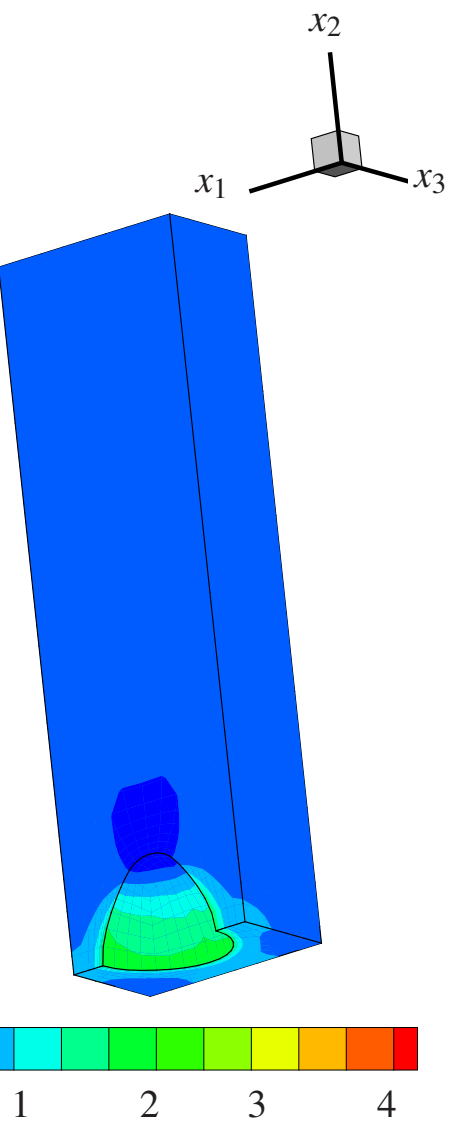

Fig. 14. Deformed unit cell at the end of the computation for $\psi_{0}=$ $0^{\circ}$, with $\kappa_{1}=0.25, \kappa_{3}=0.5$ and $R_{0} / D_{0}=0.35$. 